\title{
FIRST NEW SOLAR MODELS WITH OPAS OPACITY TABLES
}

\author{
M. Le Pennec ${ }^{1}$, S. Turck-Chièze ${ }^{1}$, S. Salmon ${ }^{1}$, C. Blancard ${ }^{2}$, P. Cossé $^{2}$, G. Faussurier ${ }^{2}$, And G. Mondet ${ }^{2}$ \\ ${ }^{1}$ CEA/IRFU/Service d'Astrophysique, CE Saclay, F-91191 Gif sur Yvette, France \\ ${ }^{2}$ CEA, DAM, DIF, F-91297 Arpajon, France \\ Received 2015 July 31; accepted 2015 October 15; published 2015 November 10
}

\begin{abstract}
Stellar seismology appears more and more as a powerful tool for a better determination of the fundamental properties of solar-type stars. However, the particular case of the Sun is still challenging. For about a decade now, the helioseismic sound-speed determination has continued to disagree with the standard solar model (SSM) prediction, questioning the reliability of this model. One of the sources of uncertainty could be in the treatment of the transport of radiation from the solar core to the surface. In this Letter, we use the new OPAS opacity tables, recently available for solar modeling, to address this issue. We discuss first the peculiarities of these tables, then we quantify their impact on the solar sound-speed and density profiles using the reduced OPAS tables taken on the grids of the OPAL ones. We use the two evolution codes, Modules for Experiments in Stellar Astrophysics and Code Liégeois d'Evolution Stellaire, that led to similar conclusions in the solar radiative zone. In comparison to commonly used OPAL opacity tables, the new solar models are computed for the most recent photospheric composition with OPAS tables and present improvements to the location of the base of the convective zone and to the description of the solar radiative zone in comparison to the helioseismic observations, even if the differences in the Rosseland mean opacity do not exceed $6 \%$. We finally carry out a comparison to a solar model computed with the OP opacity tables.
\end{abstract}

Key words: atomic processes - opacity - plasmas - stars: evolution - stars: interiors

\section{THE SOLAR RADIATIVE ZONE IN QUESTION}

The space missions ESA's CoRoT (Baglin et al. 2006) and NASA's Kepler (Gilliland et al. 2010) have already provided thousands of seismic observations of solar-like stars. This new investigation improves the knowledge of their fundamental properties (mass, radius) with the help of scaling relations (Chaplin \& Miglio 2013 and references therein). The next effort concentrates on getting insight into their interior with the help of asteroseismology. However, most of the stellar evolution codes use the same physics inputs. It is thus important to assess the validity of these inputs to get the best scientific return of such space missions.

The Sun is a necessary test case for that purpose. The solar revised CNO photospheric composition (Asplund et al. 2005) revealed that the solar sound speed, predicted by a standard solar model (SSM), is significantly different from the one obtained seismically from the $\mathrm{SOHO}$ satellite or from ground networks in the radiative zone. The differences appeared largely greater than the seismic error bars deduced with the space GOLF+MDI instruments (Turck-Chièze et al. 2001, 2004; Bahcall et al. 2005). Then, the detailed composition of the Sun was reexamined by different groups (Caffau et al. 2008; Asplund et al. 2009), but the discrepancy between the two sound-speed profiles continues to be puzzling (TurckChièze \& Couvidat 2011; Turck-Chièze et al. 2011b; Basu et al. 2014). It reaches nearly $1 \%$ on the sound speed, which is determined with a precision of $10^{-4}$ that seems difficult to attribute only to the dynamical processes (direct effect of rotation or magnetic field), which are often not included in the equations describing theoretical models.

Several hypotheses have been suggested and some of them have been quantified.

1. An incorrect understanding of the inner composition in part due to some not well-known elements and in part to insufficient treatment of the microscopic diffusion (Basu \& Antia 2008; Basu et al. 2014).

2. Insufficient knowledge of the energetic balance. An upper limit of $5 \%$ for the possible energy difference between the energy produced by the nuclear reaction rates and the release of energy at the surface of the Sun has been estimated (see Turck-Chièze \& Lopes 2012, Table 3) in using both neutrinos and seismology. If a difference exists, it could be attributed to some dynamical components not present in the energy equation of the stellar structure (Turck-Chièze 2015). This idea could be checked with a very precise measurement of the pp or pep neutrino flux.

In this Letter, we explore another hypothesis stating that the current description of the energy transport by photons is not sufficiently accurate for the interpretation of the helio- and asteroseismic observations. If this is the case, both the use of the Rosseland mean opacity values in stellar equations and the treatment of the microscopic diffusion in the radiative zone would be affected.

The available opacity tables, OPAL (Iglesias \& Rogers 1996) and OP (Seaton \& Badnell 2004), were provided more than 10 years ago. We explore in this Letter how new opacity calculations performed with current computer resources modify the solar internal thermodynamical quantities. This first estimate uses the new, recently available OPAS tables (Blancard et al. 2012; Mondet et al. 2015).

\section{THE OPAS CALCULATIONS}

A new generation of opacity codes is currently under development to improve the interpretation of stellar observations in the field of helio- and asteroseismology. One can mention the ATOMIC calculations performed at Los Alamos (Colgan et al. 2013), the SCO-RCG ones performed by a CEA 


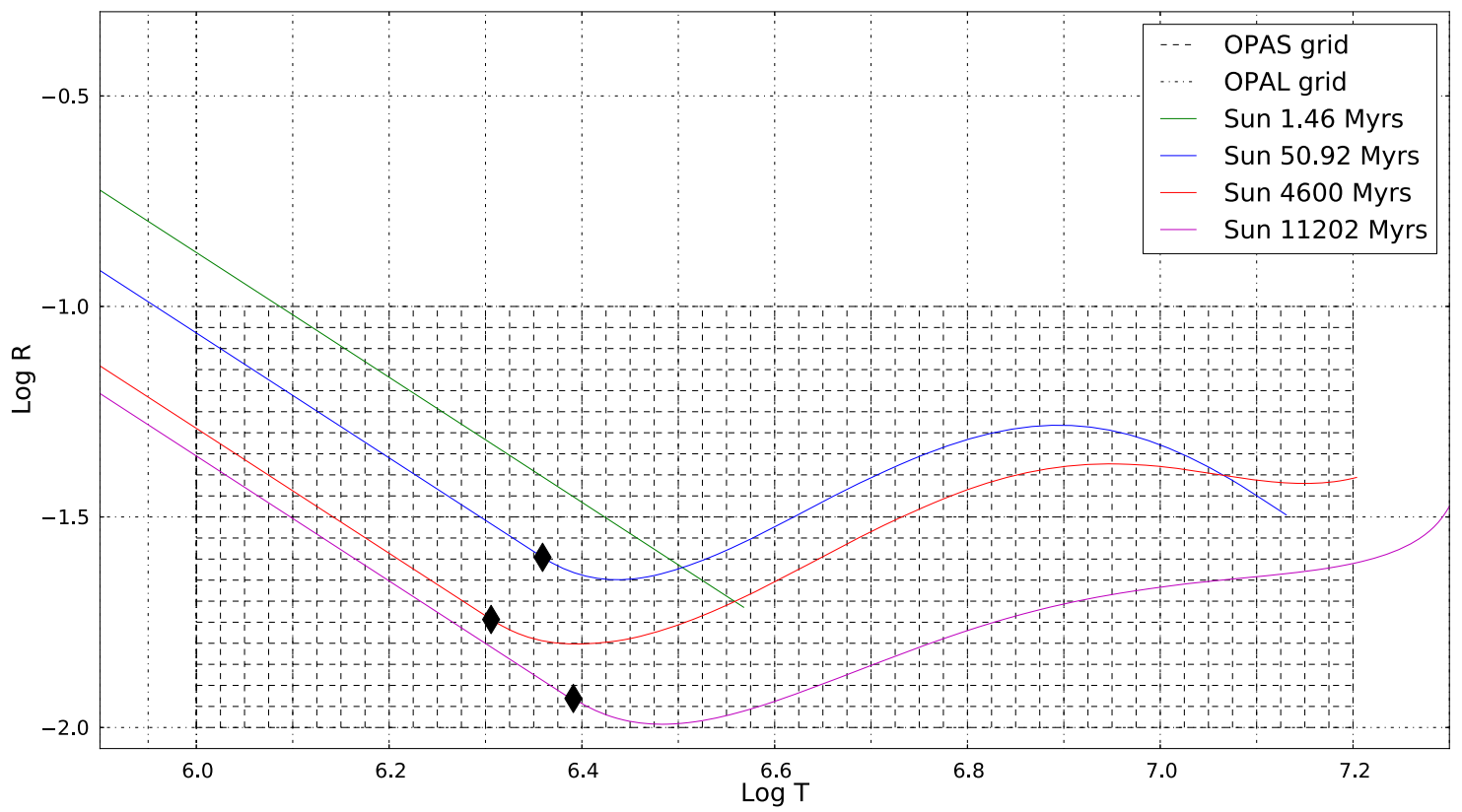

Figure 1. OPAL and OPAS opacity meshes, superimposed on the solar path at different ages (continuous line). The diamond symbol marks the transition between the radiative and the convective zone.

team (Porcherot et al. 2011), and the OPAS ones performed by another CEA team (Blancard et al. 2012). Some outputs of these codes have been compared to a new generation of opacity experiments performed at LULI2000 (Turck-Chièze et al. 2011a, 2013, 2015) and on the $Z$ machine of La Sandia (Bailey et al. 2015).

The OPAS code is dedicated to radiative opacity calculations of plasmas in local thermodynamic equilibrium. It is based on a detailed configuration approach (Blancard et al. 2012). The monochromatic opacity is evaluated as the sum of four different contributions involving the diffusion process, freefree, bound-free, and bound-bound absorption processes. The bound-bound opacity is calculated by combining different approximations to take into account the level structure of configurations. Statistical or detailed methods are used to describe the transitions connecting a couple of configurations. The detailed method is based on an extensive line accounting performed in the full intermediate coupling. The bound-free opacity is evaluated using configuration-average distorted wave calculations. The free-free opacity is obtained by interpolating between the Drude-like opacity and the opacity derived from the Kramers formula including a Gaunt factor and an electron degeneracy effect correction to improve the accuracy of opacities into the complex regime where plasma and manybody effects can be important. Photon scattering by free electrons includes some collective effects as well as relativistic corrections. The different approximations and their impact on the Rosseland mean value tables are discussed (see Mondet et al. 2015 for details, and the tables are available through this reference).

\section{DESCRIPTION OF THE OPAS TABLES}

The OPAS opacity calculations are tabulated in $\log _{10} T$ and $\log _{10} R$, like the OPAL tables, where $\log _{10} R=\log _{10} \rho-3 * \log _{10} T+18$. For reference, the OPAL tables cover $\log _{10} R$ from -8 to 1 with steps of 0.5 and $\log _{10} T$, from 3.75 to 6 with steps of 0.05 , from 6 to 8.1 with steps of 0.1 , and from 8.1 to 8.7 by steps of 0.2 .

The new OPAS tables are specifically dedicated to the study of the Sun and solar-like stars. Therefore, they have been computed with thinner grids on $\log _{10} T, \log _{10} R$, and $Z$. Consequently, for resources reasons, they are presently reduced to $\log _{10} T$ from 6 to 7.2 with steps of 0.025 and $\log _{10} R$ from -2 to -1 with steps of 0.05 as shown in Figure 1 where the paths of the Sun at different ages are represented together with the locations of the base of the convective zone. Moreover, the $Z$ grids also have been increased to better adapt to the present solar composition; $Z=0.015$ has been added along with some interpolations for 0.013 and 0.017 .

Figure 2 recalls the contributions of the most important heavy elements to the global opacity (including $\mathrm{H}$ and $\mathrm{He}$ ). This figure has been realized with OP opacity calculations, as monochromatic calculations are available for the different elements. The temperature grids corresponding to OPAL (blue circles) and OPAS (red circles) are also indicated. As one can see, each elementary contribution has a specific shape, but a spline interpolation through the OPAL (or OP) tables with only $7-8$ points in temperature in the whole radiative zone of the Sun could produce a smoothing effect that does not allow one to explore the whole potentiality of the seismic results. Therefore, the OPAS tables have been designed to significantly improve the interpolation procedure for trying to extract some inner composition signatures from seismology (currently, for the most past, for the Sun), as it was mentioned as an objective before the launch of SOHO (Turck-Chièze 1992).

Indeed, the uncertainty on the sound speed is about $10^{-4}$, while its radial location uncertainty varies from $1.5 \%$ to $3 \%$ in the radius from the $\mathrm{BCZ}$ to the center. Hence, a small number of opacity points does not seem sufficient to precisely probe the composition of this region since the Rosseland mean values are significantly dependent on the ionization state of each element (see Turck-Chièze et al. 1993). The OPAL Rosseland mean opacity varies between two consecutive points of the grid by 


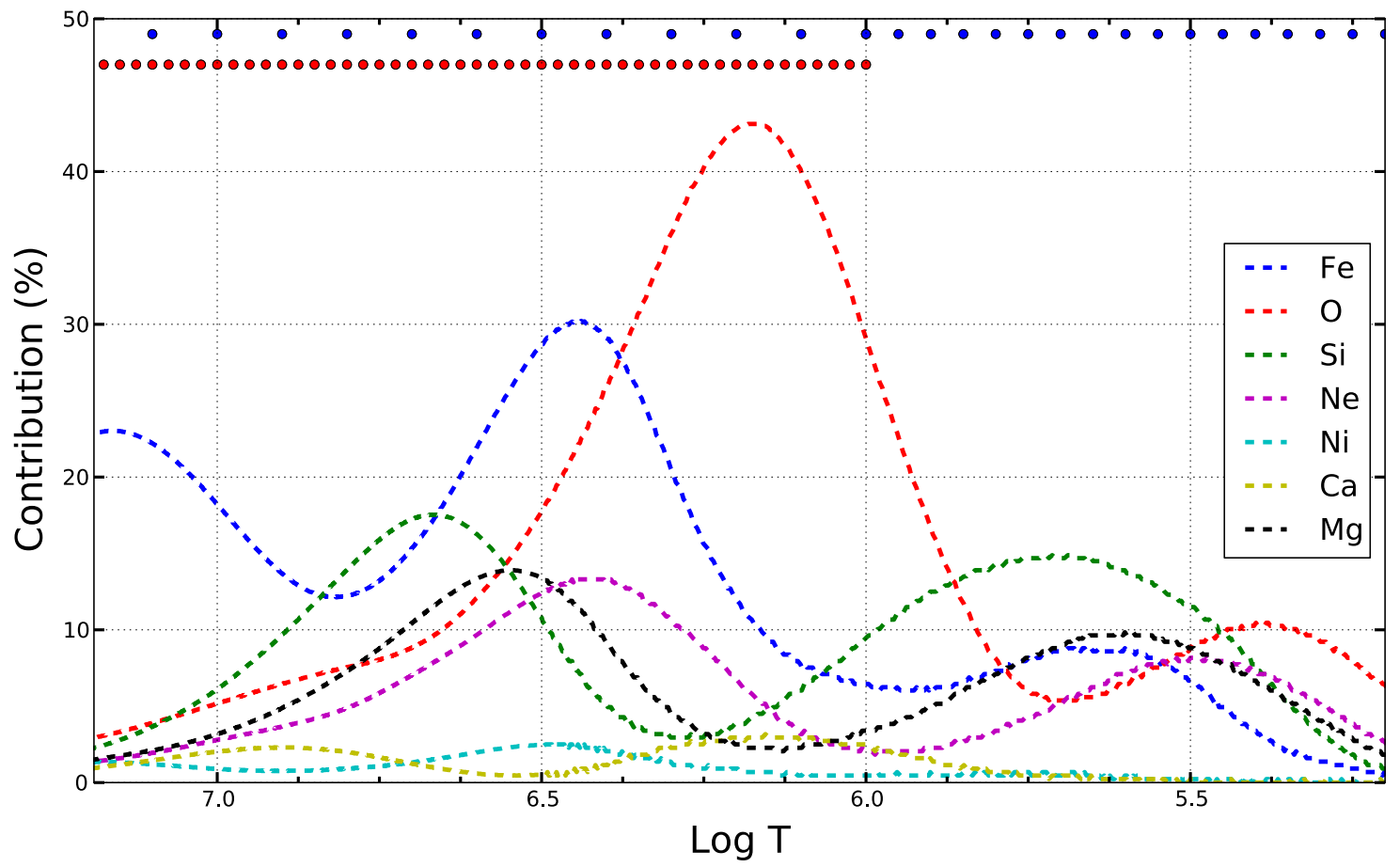

Figure 2. Relative contribution of the most important heavy elements to the total Rosseland mean opacity (including $\mathrm{H}$ and $\mathrm{He}$ ) for the internal conditions of the present Sun and the composition from Asplund et al. (2009), using OP opacities. OPAS (red circles) and OPAL (blue circles) grids in temperature are indicated in the upper part of the diagram.

Table 1

Comparison between Solar MESA and CLES Models Including OPAL, OPAS, or OP Opacity Calculations and the Most Recent Composition (Asplund et al. 2009)

\begin{tabular}{lccccc}
\hline \hline & $\begin{array}{c}\text { MESA- } \\
\text { OPAL }\end{array}$ & $\begin{array}{c}\text { MESA- } \\
\text { OPAS }\end{array}$ & $\begin{array}{c}\text { CLES- } \\
\text { OPAL }\end{array}$ & $\begin{array}{c}\text { CLES- } \\
\text { OPAS }\end{array}$ & CLES-OP \\
\hline$Y_{0}$ & 0.2654 & 0.2611 & 0.2681 & 0.2636 & 0.2666 \\
\hline$\alpha$ & 1.77 & 1.79 & 1.75 & 1.76 & 1.76 \\
\hline$(\mathrm{Z} / X)_{S}$ & 0.01816 & 0.01815 & 0.01810 & 0.01810 & 0.01810 \\
\hline$R_{\mathrm{CZ}}\left(R_{\odot}\right)$ & 0.729 & 0.723 & 0.724 & 0.719 & 0.723 \\
\hline$T_{\mathrm{C}}(\mathrm{K})$ & $15.5510^{6}$ & $15.5410^{6}$ & $15.5510^{6}$ & $15.5410^{6}$ & $15.5210^{6}$ \\
\hline
\end{tabular}

Note. $Y_{0}$ is the initial helium, $\alpha$ the MLT value, $Z / X_{S}$ the surface metallic/ hydrogen ratio at the present age, $R_{\mathrm{CZ}}$ the position of the base of the convective zone, and $T_{\mathrm{C}}$ the central temperature.

about $25 \%$ with a change of more than a factor of 10 between the center to the $\mathrm{BCZ}$ of a solar model. The fine mesh of OPAS presents only $6 \%$ of the Rosseland mean opacity variation between two consecutive points of the grids, so the interpolation between points (when introduced in the computation of solar models) will be more accurate. Consequently, OPAS tables will have the potential of interpreting with a better sensitivity changes of slope in the sound-speed profile due to the different opacity processes behavior (see Section 2) available from different element contributions. These tables will also have the potential to develop the inversion of composition inside the radiative zone, as was possible for the equation of state in the subsurface layers of the Sun (Basu \& Christensen-Dalsgaard 1997).
First, one needs to see how the absolute differences between OPAS and the most commonly adopted tables act on the solar model. This is why in this Letter we compare the structures of solar models computed with OPAS, OPAL, and OP tables by using the same opacity mesh in each case, i.e., adopting the OPAL standard one (see the beginning of this section). In doing so, we do not introduce any adding effect of interpolation that could be difficult to dissociate from physical processes. We use in that aim two evolution codes popular in the asteroseismic community.

From Mondet et al. (2015), we know that the OPAS calculations do not differ by more than $10 \%$ from the OPAL ones. In the present study, no more than $6 \%$ differences are observed between OPAS and OPAL calculations for solar conditions, so one needs to be cautious regarding our conclusions.

\section{NEW SOLAR MODELS USING THE OPAS TABLES}

In this section, we compare the SSM computed for the most recent composition (Asplund et al. 2009) with two different stellar evolution codes, Modules for Experiments in Stellar Astrophysics (MESA) and Code Liégeois d'Evolution Stellaire (CLES). We compare first the impact between the use of OPAS and OPAL tables, as OPAL tables are considered the best effort in opacities done for solar and solar-type stellar applications. The use of the two codes guarantees that the observed effects are really due to the new physics taken into account in the opacity calculations. This precaution is necessary as the differences between the two tables are not so large.

\subsection{The MESA Characteristics}

MESA (Paxton et al. 2011; Paxton et al. 2013, 2015) is a recent stellar evolution code performed for extensive use in the 

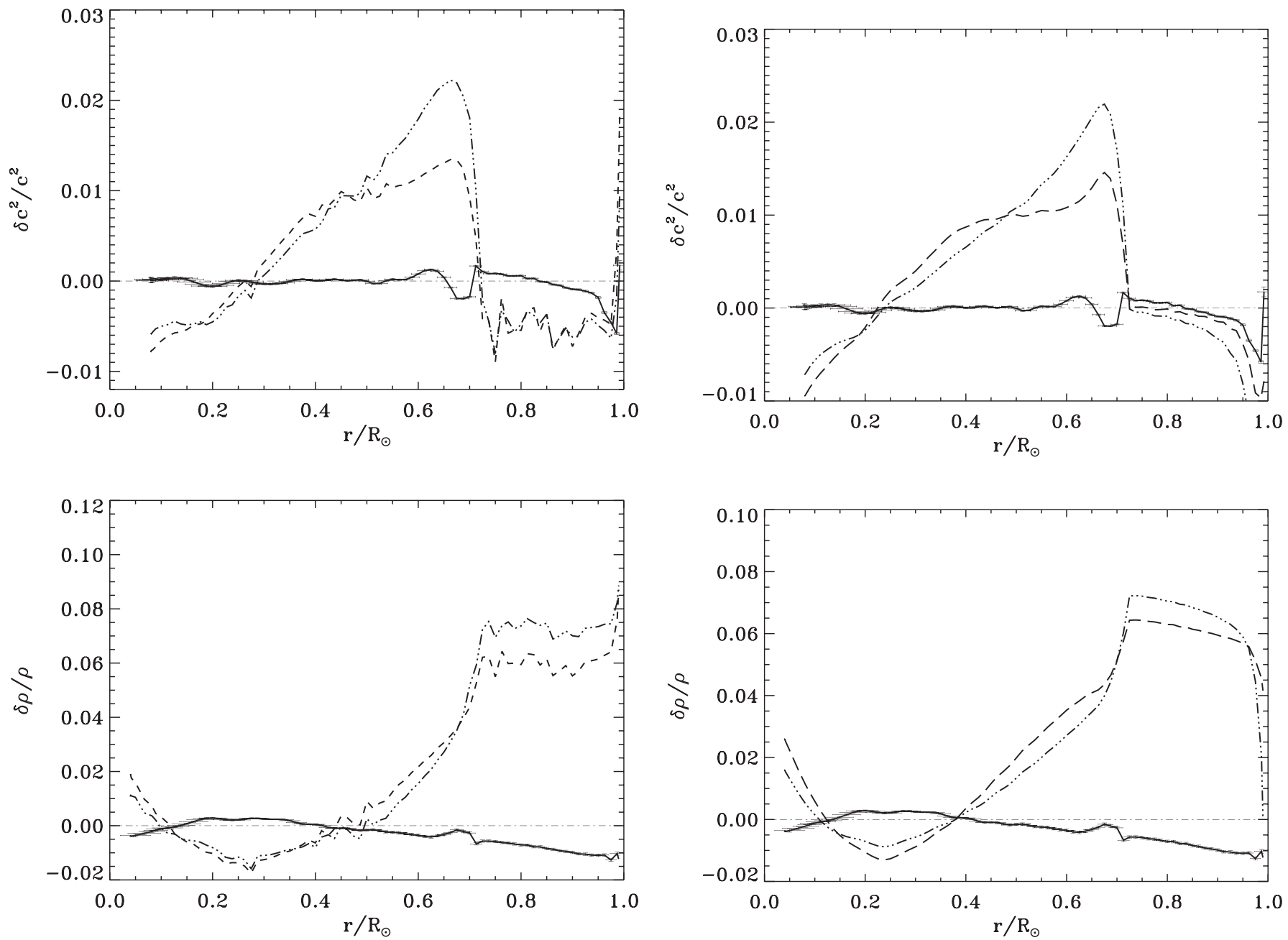

Figure 3. Left: difference between the observed squared sound-speed and density profiles with those obtained with an SSM model of MESA using OPAL (-..- line) or OPAS (-- line). Right: idem for an SSM model performed with CLES. The full line corresponds to a seismic model; the associated error bars are extracted from the inversion done using GOLF+MDI on board SOHO observations (see Turck-Chièze \& Lopes 2012 for numbers and details).

HR diagram. This code is now largely used in the asteroseismic community due to its reliability and its extensive access to a large range of mass and evolution stages. The rapid progress in the introduction of the physical inputs due to its international use makes it very attractive for a lot of astrophysical applications.

In the present study, we use version 4906 of the code and adopt the following physics inputs: the MLT theory (BöhmVitense 1958), the OPAL EOS (Rogers \& Nayfonov 2002), and OPAL opacity tables extended to low $T$ and $\rho$ (Ferguson et al. 2005). Nuclear reactions are taken from NACRE (Angulo et al. 1999), and the microscopic diffusion of all the elements uses the subroutine of Thoul et al. (1994). The MESA atmosphere model (Paxton et al. 2011) comes from tables performed by Castelli \& Kurucz (2003), using the solar composition of Grevesse \& Noels (1993).

\subsection{The CLES Characteristics}

The stellar evolution code CLES (Scuflaire et al. 2008) has been developed mainly for main-sequence studies and seismic interpretation and, for instance, has been compared in detail with the CESAM code (see detailed comparisons in Montalbán et al. 2008). An additional smoothing of the opacity tables before their use in the evolution code is an option in CLES. As we observe that it can artificially reduce the values of the opacity, we do not include such treatment in the present study. We use the same physics input as for the MESA computations, except that the treatment of the microscopic diffusion only considers three elements: $\mathrm{H}, \mathrm{He}$, and $\mathrm{Fe}$ (all elements heavier than $\mathrm{He}$ are treated as $\mathrm{Fe}$ ). The code uses interpolation in models of atmosphere (see Kurucz 1998) and performs a smooth junction between interior and atmosphere at $T=T_{\text {eff }}$ of the model, with the same limitation as MESA.

\subsection{Use of OPAS Tables in the Stellar Evolution Codes}

We have built several calibrated solar models with CLES and MESA using OPAL and OPAS tables. In the second case and since OPAS tables extend over a limited range of $\log _{10} T$ and $\log _{10} R$ values, at each mesh point of the OPAL tables where there is an existing OPAS calculation, the OPAL opacity value is replaced by the corresponding OPAS value. The OPAL values are adopted for points outside the OPAS domain, but we note that there is no transition in tables due to the mixing of OPAL and OPAS information, as the OPAS tables cover the whole solar radiative zone study. 

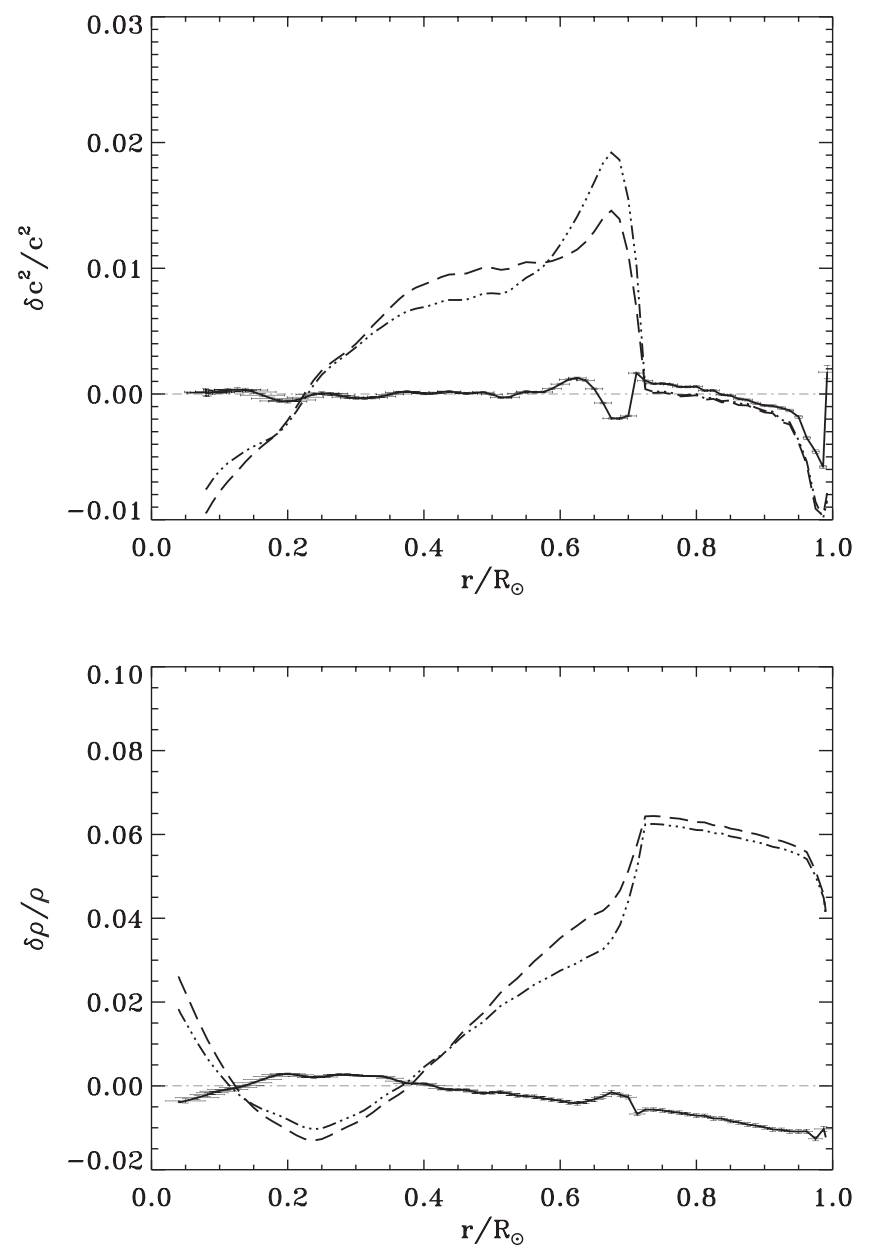

Figure 4. Difference between the observed squared sound-speed and density profiles (Turck-Chièze \& Lopes 2012) with those obtained with an SSM model of CLES and using OP opacity $(-\ldots-$ line) or OPAS $(---$ line). Same comments as in Figure 3.

Table 1 summarizes the quantities of interest for the calibrated solar models that we have computed. One should note that, in both cases, the base of the convective zone becomes closer to the seismic results $\left(0.713 \pm .001 R_{\odot}\right.$; Basu \& Antia 1997) with the OPAS tables. The initial helium abundance also decreases when using OPAS tables in both cases. With CLES, we have also compared the new results to a solar model using OP tables as was already done for a different solar composition (Scuflaire et al. 2008). We note the same tendencies between OP and OPAS than between OPAL and OPAS for the position of the base of the convective zone and for the initial helium.

\section{SOUND-SPEED AND DENSITY PROFILES COMPARED TO HELIOSEISMIC RESULTS}

We have extracted the solar sound speed and density from the previous models, and we compare them to the seismic observations (see all the numbers in Turck-Chièze \& Lopes 2012).

\subsection{Comparison between Models Using $O P A L$ and OPAS Opacities}

Figure 3 shows a clear reduction of the difference between the SSM squared sound-speed or density profile and the observed seismic values along one-third of the radiative zone below the base of the convective zone when one uses the OPAS values in the OPAL tables. The same effect is observed for the two evolutionary codes and can be directly attributed to the change of opacities.

The observed improvement could be attributed to more complete opacity calculations of iron, nickel, and several other low-abundant-element (with high atomic numbers) contributors to the Rosseland mean OPAS values. Indeed, near the base of the convection zone, bound-bound processes are important for these elements. Even if it is difficult to conclude without a detailed comparison of the spectra, it is important to recall that $6 \%$ of the mean value could come from $30 \%$ to $40 \%$ differences on some specific elements (see Blancard et al. 2012). Moreover, the difference in absolute values of the position of the $\mathrm{BCZ}$ could originate from the way the opacities are used (smoothing or not smoothing of the opacities) in the two codes and on the difference in the treatment of the microscopic diffusion. This point will be studied in detail in a more thorough paper.

On the contrary, in the nuclear region and slightly above it, the agreement is slightly worse and the central temperature slightly reduced, as shown in Table 1 , due to a reduction by less than $5 \%$ of the Rosseland mean values of OPAS compared to OPAL ones (as shown on Figure 5 by Mondet et al. 2015). The reasons have not been studied in detail, but a check of the reliability of these calculations would be useful. Some experimental validation to study the plasma effects has been already studied (Le Pennec et al. 2015).

\subsection{Comparison between Models Using $O P$ and OPAS Opacities}

One can see in Figure 4 that the improvements for models computed with CLES, passing from OP to OPAS tables, seem much smaller. Nevertheless, Table 1 shows the same progress for the position of the base of the convective zone. In fact, OPAS monochromatic opacity calculations differ from OP calculations in the description of the Stark profile of the Healpha line (Blancard et al. 2012). The width is greater in OP calculations, and this effect increases with $Z$. Indeed, oxygen, neon, magnesium, and silicium are affected by this effect with resulting larger opacities for these elements in the case of OP calculations. On the contrary, in the case of iron, due to the greater number of considered excited states, OPAS calculations are greater than OP ones. Therefore, as the differences in oxygen and iron opacities are in the opposite sign, the recent progress performed by the new generation of opacity codes is not clearly visible, but the surprising result on the $Z$ pinch experiment does not favor the OP opacity calculations on iron as compared to the OPAS ones (Bailey et al. 2015).

\section{CONCLUSION AND PERSPECTIVES}

New refined opacity tables are now available for the modeling of the Sun and solar-like stars (Mondet et al. 2015). In this Letter, we show the physical change obtained in using OPAS tables in OPAL or OP grids with the same mesh. These improved calculations present opacity differences with OPAL of no more than $\pm 5 \%-6 \%$ in the conditions used in the present study. Such changes already reduce the differences with the seismic observations when compared to the use of OPAL tables, both for the base of the 
convective zone and for the sound-speed profile in the radiative region, which could be attributed to a more complete treatment of the bound-bound processes of the iron group elements. The progress in comparison with OP is also shown, but it is largely reduced due a compensation effect between iron and oxygen. Nevertheless, OP is not preferred to OPAS when the recent $Z$ machine experiment is taken into account.

The present study shows the direct effect of improvement in the opacity calculations for some elements of the iron group. Interest in the OPAS tables goes beyond the present study, as the fine grids in $\log _{10} T, \log _{10} R$, and $Z$ will improve the interpolation through the tables for Sun and solar-like stars. The fine meshes of OPAS will be used to try to extract some specific signatures of the deep composition of the Sun. This work is in progress and a more complete study using the potential of the fine meshes of OPAS will be discussed in a more detailed paper (S. Salmon et al. 2015, in preparation). The present results strongly encourage complementary experimental studies on high energy density laser facilities, both on iron and oxygen (Keiter et al. 2013; Le Pennec et al. 2015).

This work has been done in the framework of the French ANR OPACITY. We would also like to thank J. Montalbán for her great expertise in the use of the CLES code. We thank also the referees for their judicious remarks that lead to an improved Letter.

\section{REFERENCES}

Angulo, C., Arnould, M., Rayet, M., et al. 1999, NuPhA, 656, 3 Asplund, M., Grevesse, N., Sauval, A. J., Allende Prieto, C., \& Kiselman, D. 2005, A\&A, 435, 339

Asplund, M., Grevesse, N., Sauval, A. J., \& Scott, P. 2009, ARA\&A, 47, 481

Baglin, A., Michel, E., Auvergne, M., \& COROT team 2006, in Proc. SOHO

18/GONG 2006/ HELAS I 624, Beyond the Spherical Sun (ESA SP-264; Noordwijk: ESA), 34

Bahcall, J. N., Serenelli, A. M., \& Basu, S. 2005, ApJL, 621, L85

Bailey, J. E., Nagayama, T., Loisel, G. P., et al. 2015, Natur, 517, 56
Basu, S., \& Antia, H. M. 1997, MNRAS, 287, 189

Basu, S., \& Antia, H. M. 2008, PhR, 457, 217

Basu, S., \& Christensen-Dalsgaard, J. 1997, A\&A, 332, L5

Basu, S., Grevesse, N., Mathis, S., \& Turck-Chièze, S. 2014, SSRv, in press

Blancard, C., Cossé, P., \& Faussurier, G. 2012, ApJ, 745, 10

Böhm-Vitense, E. 1958, ZAp, 46, 108

Caffau, E., Ludwig, H.-G., Steffen, M., et al. 2008, A\&A, 488, 1031

Castelli, F., \& Kurucz, R. L. 2003, in IAU Symp. 210, Modelling of Stellear Atmospheres, ed. N. Piskunov, W. W. Weiss \& D. F. Gray (San Franscisco, CA: ASP), A20

Chaplin, W. J., \& Miglio, A. 2013, A\&A, 51, 353

Colgan, J., Kilcrease, D. P., Magee, N. H., et al. 2013, HEDP, 9, 369

Ferguson, J. W., Alexander, D. R., Allard, F., et al. 2005, ApJ, 623, 585

Gilliland, R. L., Brown, T. M., Christensen-Dalsgaard, J., et al. 2010, PASP, 122,131

Grevesse, N., \& Noels, A. 1993, in Origin and Evolution of the Elements, ed. N. Prantzos, E. Vangioni-Flam \& M. Cassé (Cambridge: Cambridge Univ. Press), 15

Iglesias, C. A., \& Rogers, F. J. 1996, ApJ, 464, 943

Keiter, P. A., Mussack, K., \& Klein, S. R. 2013, HEDP, 9, 319

Kurucz, R. L. 1998, HiA, 11, 646

Le Pennec, M., Ribeyre, X., Ducret, J.-E., \& Turck-Chièze, S. 2015, HEDP, 17,163

Mondet, G., Blancard, C., Cossé, P., \& Faussurier, G. 2015, ApJS, 220, 2

Montalbán, J., Lebreton, Y., Miglio, A., et al. 2008, Ap\&SS, 316, 219

Paxton, B., Bildsten, L., Dotter, A., et al. 2011, ApJS, 192, 3

Paxton, B., Cantiello, M., Arras, P., et al. 2013, ApJS, 208, 4

Paxton, B., Marchant, P., Schwab, J., et al. 2015, ApJS, 220, 15

Porcherot, Q., Pain, J.-C., Gilleron, F., \& Blenski, T. 2011, HEDP, 7, 234

Rogers, F. J., \& Nayfonov, A. 2002, ApJ, 576, 1064

Scuflaire, R., Théado, S., Montalbán, J., et al. 2008, Ap\&SS, 316, 83

Seaton, M. J., \& Badnell, N. R. 2004, MNRAS, 354, 457

Thoul, A. A., Bahcall, J. N., \& Loeb, A. 1994, ApJ, 421, 828

Turck-Chièze, S. 1992, NuPhS, 28, 116

Turck-Chièze, S. 2015, JPhCS, in press

Turck-Chièze, S., \& Couvidat, S. 2011, RPPh, 74, 086901

Turck-Chièze, S., Couvidat, S., Kosovichev, A. G., et al. 2001, ApJL, 555, L69

Turck-Chièze, S., Couvidat, S., Piau, L., et al. 2004, PhRvL, 93, 211102

Turck-Chièze, S., Däppen, W., Fossat, E., et al. 1993, PhR, 230, 57

Turck-Chièze, S., Gilles, D., Le Pennec, M., et al. 2013, HEDP, 9, 473

Turck-Chièze, S., Le Pennec, M., Ducret, J.-E., et al. 2015, CEA internal report

Turck-Chièze, S., Loisel, G., Gilles, D., et al. 2011a, Ap\&SS, 336, 103

Turck-Chièze, S., \& Lopes, I. 2012, RAA, 12, 1107

Turck-Chièze, S., Piau, L., \& Couvidat, S. 2011b, ApJL, 731, L29 